\title{
Pensamento computacional sob a perspectiva de licenciandos em computação
}

\author{
Rozelma Soares de França ${ }^{1,2}$, Patrícia Cabral de Azevedo Restelli Tedesco ${ }^{1}$ \\ ${ }^{1}$ Centro de Informática - Universidade Federal de Pernambuco (UFPE) \\ Recife, PE - Brasil \\ ${ }^{2}$ Departamento de Educação - Universidade Federal Rural de Pernambuco (UFRPE) \\ Recife, PE - Brasil \\ \{rsf2, pcart\}@cin.ufpe.br
}

\begin{abstract}
Resumo. A literatura da área de Educação em Computação aponta para a necessidade de integrar o Pensamento Computacional (PC) no currículo escolar, tendo os estudantes uma compreensão dos fundamentos da Ciência da Computação, não limitando-se ao manuseio de tecnologias. Nesse contexto, este artigo busca identificar o entendimento que licenciandos em Computação possuem acerca do PC, uma que vez que a maneira como apropriam-se dessa habilidade interferirá no seu modo de ensinar. A partir de um estudo realizado em uma universidade pública no Brasil pôde-se identificar que, em geral, a compreensão de PC que os futuros professores possuem é condizente com a literatura da área. Em particular, foi identificado que os conceitos Algoritmo, Automação e Abstração são os que eles percebem explorar com maior recorrente na resolução de problemas do dia a dia, em detrimento do uso de Paralelismo e Simulação.
\end{abstract}

\section{Introdução}

Em um mundo cada vez mais tecnológico, é necessário que os estudantes tenham um claro entendimento dos princípios e práticas da Ciência da Computação (CC), independente de seus campos de estudo e atuação (CSTA, 2011). O acesso ao conhecimento dos fundamentos de CC pode auxiliar a utilizar tecnologias de maneira mais consciente, além de apoiá-los na criação de sistemas de computação para melhorar a qualidade de vida das pessoas (ibid). A $\quad$ CC na educação básica deve perpassar pela aprendizagem de fundamentos da área, não se limitando à operacionalização de tecnologias. Nesse contexto, Wing (2006) justifica o ensino desta habilidade, que denominou de Pensamento Computacional (PC).

A International Society for Technology in Education (ISTE) em colaboração com a Computer Science Teachers Association (CSTA) e a National Science Foundation (NSF) elaboraram uma definição operacional de PC, segundo a qual ele é um processo de resolução de problemas que inclui, mas não se limita, às seguintes características: $i$ ) formular problemas de modo que seja possível usar o computador e outras ferramentas para ajudar a resolvê-los; ii) organizar e analisar dados, de forma lógica; iii) representar dados através de abstrações, tais como modelos e simulações; $i v$ ) automatizar soluções através do pensamento algorítmico; $v$ ) identificar, analisar e implementar as soluções possíveis com o objetivo de conseguir a combinação mais eficiente e eficaz de etapas e recursos; e $v i$ ) generalizar e transferir esse processo de resolução de problemas para uma grande variedade de problemas. Os seguintes conceitos estão embutidos nessa definição: coleta, análise e representação de dados, decomposição de problemas, abstração, algoritmos e procedimentos, automação, simulação e paralelismo (ISTE; CSTA; NSF, 2011). 
VI Congresso Brasileiro de Informática na Educação (CBIE 2017)

Anais do XXIII Workshop de Informática na Escola (WIE 2017)

Nesse contexto, alguns desafios são identificados no cenário brasileiro. Um deles perpassa pelo currículo escolar que não considera a CC como uma de suas disciplinas; apesar de haver uma conquista da sociedade publicada na terceira versão da Base Nacional Comum Curricular (BNCC) ${ }^{1}$ que diz que uma das dez competências gerais que devem perpassar os componentes curriculares da educação básica diz respeito à cultura digital, como pode ser observado em sua descrição:

\footnotetext{
"Utilizar tecnologias digitais de comunicação e informação de forma crítica, significativa, reflexiva e ética nas diversas práticas do cotidiano (incluindo as escolares) ao se comunicar, acessar e disseminar informações, produzir conhecimentos e resolver problemas." (MEC, 2017).
}

Tal problema é agravado por perceber-se que não há um manifesto da sociedade brasileira clamando pela introdução da CC no currículo escolar. Isto sugere que, em geral, não há uma consciência sobre a importância de tal ciência; sendo esta consciência tida, em sua maioria, por atuantes na Educação em Computação. Há, por outro lado, a crescente oferta do curso de Licenciatura em Computação (LC) no país, conforme apontado pelas Estatísticas da Educação Superior em Computação de $2015^{2}$, o qual versa, principalmente, a formação de professores para o ensino da CC na educação básica.

Diante desse cenário, diferentes problemas de pesquisa podem ser investigados, e o objetivo deste trabalho é identificar como o pensamento computacional é compreendido por professores da área que atuarão na educação básica. É oportuno investigar esse problema, porque não há a definição de competências e habilidades de Computação na BNCC, tal qual as especificadas no CSTA K-12 Computer Science Standards ${ }^{3}$. Pontua-se, por outro lado, que recentemente referenciais de formação em Computação para educação básica foram elaborados pela SBC para serem implementados em escolas brasileiras. Diante desse cenário, diferentes fontes poderão ser consultadas pelos professores que, em suas práticas pedagógicas, poderão dar mais ênfase ao manuseio de recursos tecnológicos, em detrimento do ensino dos princípios fundamentais da Computação. A maneira como esses profissionais relacionam-se com essa habilidade computacional poderá, pois, interferir na escolha de recursos e métodos que irão nortear sua prática em sala de aula.

O restante do artigo está organizado como segue: na seção 2 são apresentados o embasamento teórico da pesquisa e trabalhos correlatos; a seção 3 descreve o método de pesquisa empregado para alcançar o objetivo desta investigação; na seção 4 são expostos e discutidos os resultados alcançados com um estudo de caso realizado com estudantes de um curso de Licenciatura em Computação; e, por fim, a seção 5 traz as considerações finais acerca de todo o trabalho.

\section{Fundamentação teórica e trabalhos relacionados}

Nesta seção, PC será definido e discutido como uma habilidade necessária a todos. Também, serão apresentados o curso que forma professores para o ensino de Computação na educação básica e trabalhos que buscam identificar a percepção de PC tida por profissionais da área.

\subsection{Pensamento computacional}

O pensamento computacional pode ser definido como o processo cognitivo utilizado pelo ser

\footnotetext{
${ }^{1}$ Disponível em <http://basenacionalcomum.mec.gov.br/images/BNCC_publicacao.pdf>. Acesso em 01 de jun. 2017.

${ }^{2}$ Disponível em <http://www.sbc.org.br/documentos-da-sbc/category/133-estatisticas >. Acesso em 19 de mar. 2017.

${ }^{3}$ Disponível em $<$ https://www.csteachers.org/page/CSTA Standards> . Acesso em 19 de mar. 2017.
} 
VI Congresso Brasileiro de Informática na Educação (CBIE 2017)

Anais do XXIII Workshop de Informática na Escola (WIE 2017)

humano para encontrar algoritmos para resolver problemas (WING, 2006). Ele é a base da CC, podendo ser aplicado na resolução de problema de outras áreas, como Jornalismo, Biologia e Matemática. Apesar do ensino de PC não compor o currículo da educação básica no Brasil, é possível identificar que diversas iniciativas têm sido realizadas no país, reportadas em conferências como o Workshop de Ensino em Pensamento Computacional, Algoritmos e Programação ${ }^{4}$, o Workshop sobre Educação em Computação ${ }^{5}$, o Workshop de Informática na Escola $^{6}$ e o Simpósio Brasileiro de Informática na Educação ${ }^{7}$. Além disso, a Sociedade Brasileira de Computação (SBC) chancelou recentemente projetos considerados inovadores e que buscam levar a Computação para crianças, adolescentes, terceira idade e públicos que não têm contato com a área na educação formal, aproximando a computação da sociedade brasileira. A distribuição geográfica dos projetos chancelados pela SBC é ilustrada na Figura 1 e, como pode ser observado, todas as regiões brasileiras têm desenvolvido ações de caráter educativo, social, cultural ou tecnológico. O detalhamento dos projetos pode ser consultado no site da $\mathrm{SBC}^{8}$.

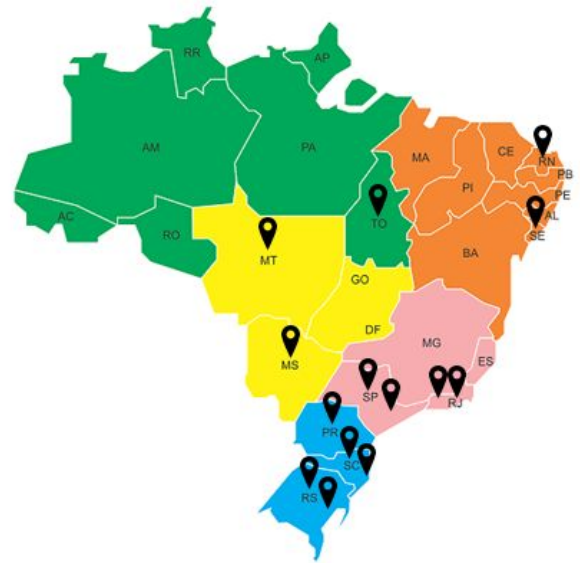

Figura 1. Estados brasileiros com projetos chancelados pela SBC

\subsection{Formação de professores de Computação}

No Brasil, diversos cursos de graduação em Computação são ofertados. São eles: Bacharelados em Ciência da Computação, em Engenharia de Computação, em Engenharia de Software, em Sistemas de Informação, além da Licenciatura em Computação, sendo este último responsável pela formação de professores de Computação para a educação básica.

De acordo com as Estatísticas da Educação Superior em Computação de 2015, há no país 103 cursos de LC, tendo uma evolução de $13.19 \%$ em comparação a 2014. Do total, 15 cursos estão localizados na região Centro-Oeste, 36 no Nordeste, 23 no Norte, 14 no Sudeste e 15 no Sul. Apesar destes dados e da importância do PC na educação básica, o ensino dessa habilidade computacional ainda não se constitui uma disciplina do currículo da educação básica brasileira, ao contrário dos Estados Unidos e de países da Europa que já têm implantado um currículo mínimo de Computação em suas escolas (CSTA, 2005).

Como destaca Nunes (2008), os cursos de LC diferem um pouco das demais licenciaturas,

\footnotetext{
${ }^{4}$ http://br-ie.org/pub/index.php/wcbie

5 http://www.lbd.dcc.ufmg.br/bdbcomp/servlet/PesquisaEvento?evento=WEI

${ }^{6} \mathrm{http}: / / \mathrm{br}$-ie.org/pub/index.php/wie

7 http://br-ie.org/pub/index.php/sbie

${ }^{8}$ http://sbc.org.br/institucional-3/chancela-sbc
} 
VI Congresso Brasileiro de Informática na Educação (CBIE 2017)

Anais do XXIII Workshop de Informática na Escola (WIE 2017)

por os licenciados deste curso adquirirem habilidades para atuarem também fora do ambiente escolar. O conhecimento de Computação, atrelado ao saber pedagógico, faz do professor de Computação único para o desenvolvimento de tecnologias da educação. O mesmo autor ainda esclarece que do ponto de vista da qualidade os cursos de LC são tão intensivos quanto os bacharelados da área de Computação e, diferentemente destes, deve-se desenvolver uma formação profissional geral, como em Banco de Dados, Sistemas Operacionais e Redes de Computadores, mas uma formação profunda em Engenharia de Requisitos e Inteligência Artificial. Ainda, os cursos de LC se completam ao trabalharem as áreas de Pedagogia, Epistemologia e Psicologia.

\subsection{Trabalhos relacionados}

Ao analisar-se a literatura sobre Educação em Computação, percebe-se que boa parte dos trabalhos é dedicada ao relato de cursos de PC para professores e estudantes da educação básica; como também à proposição ou análise de recursos, tais como jogos, para o ensino dos fundamentos da Computação.

Em outra direção, pode-se citar o estudo de Araújo et al. (2015) que buscaram identificar a percepção de PC e suas habilidades associadas sob a ótica de profissionais da Computação provenientes de bacharelados e licenciatura da área. Seus resultados sugerem que o termo PC é pouco conhecido e compreendido pelos profissionais que atuam na academia e na indústria. $\mathrm{O}$ trabalho ainda reporta que decomposição e automação são as habilidades que os pesquisados apontaram explorar com maior frequência tanto no contexto de atividades laborais como no cotidiano.

Já Bower et al. (2015) visavam identificar as atitudes e o entendimento de professores sobre pensamento computacional. Dentre os resultados reportados, os autores perceberam que enquanto muitos professores foram capazes de identificar conceitos-chave de PC, como "resolução de problemas" e "pensamento lógico", uma grande parte possui uma compreensão equivocada, associando PC ao uso básico de tecnologias.

Diferentemente dos trabalhos reportados, esta pesquisa visa identificar a percepção de PC tida por professores que atuarão no ensino dessa habilidade no Brasil, agregando valor no contexto da LC no país, onde não foi identificado a realização de investigações como esta.

\section{Método}

Objetivando identificar como futuros professores de Computação compreendem PC e quais habilidades associadas ao termo eles percebem explorar em suas atividades cotidianas, um estudo de caso foi realizado no curso de Licenciatura em Computação da Universidade Federal Rural de Pernambuco (UFRPE), buscando contribuir para tomada de decisões para melhoria da formação docente dessa licenciatura. A pesquisa realizada tem natureza qualitativa. Sua unidade de análise é constituída pelos estudantes matriculados na disciplina Estágio Supervisionado Obrigatório (ESO) ofertada no último período do curso e que oportuniza a formação em ação nos possíveis campos de atuação desses licenciandos.

\subsection{Problema de pesquisa}

Para nortear esta investigação, as seguintes perguntas de pesquisa foram definidas:

- Q1: Qual é o entendimento de futuros professores de Computação sobre pensamento computacional?

- Q2: Quais habilidades de pensamento computacional futuros professores de Computação 
VI Congresso Brasileiro de Informática na Educação (CBIE 2017)

Anais do XXIII Workshop de Informática na Escola (WIE 2017)

reconhecem explorar em atividades do dia a dia?

\subsection{Participantes}

Durante a realização da pesquisa, em 2016, sete estudantes do curso de Licenciatura em Computação da UFRPE responderam ao questionário proposto. Tais estudantes já haviam cursado disciplinas que possibilitam sua formação pela vivência de atividades pedagógicas em escolas e estavam matriculados no último ESO do curso. Tendo em vista a vivência dos licenciandos nesses componentes curriculares e por estarem no último período do curso de LC, enxergou-se neles o perfil de participantes adequado para esta pesquisa, considerando o objetivo delineado.

\subsection{Preparação da coleta de dados}

Um questionário foi elaborado para coleta de dados. Além de dados de identificação dos participantes; outras cinco perguntas compunham o instrumento, sendo que quatro delas visavam identificar: i) a consciência sobre o termo pensamento computacional; ii) sua definição; iii) estratégias pedagógicas e uso de tecnologias no ensino de PC. Tais perguntas deram suporte à resposta da Q1.

A outra pergunta foi voltada a identificar as habilidades exploradas no dia a dia dos licenciandos em Computação. Para elaborá-la, levou-se em consideração a definição operacional de PC proposta pela International Society for Technology in Education (ISTE) em colaboração com a Computer Science Teachers Association (CSTA) e a National Science Foundation (NSF), descrita na seção 1 deste artigo. No questionário, as habilidades foram descritas conforme a Figura 2. Aos participantes do estudo fora solicitado classificar cada uma delas em uma escala likert com as seguintes opções de resposta: $i$ ) nunca; $i i)$ raramente; iii) às vezes; $i v$ ) frequentemente; e $v$ ) sempre.

\begin{tabular}{|c|c|c|c|c|c|}
\hline \multicolumn{6}{|c|}{$\begin{array}{l}\text { Nas suas atividades do dia a dia, com que frequência você explora as habilidades listadas a } \\
\text { seguir? }\end{array}$} \\
\hline & Nunca & Raramente & As vezes & Frequentemente & Sempre \\
\hline \multicolumn{6}{|l|}{$\begin{array}{l}\text { Reunir dados de forma apropriada para } \\
\text { executar uma atividade especifica. }\end{array}$} \\
\hline \multicolumn{6}{|l|}{$\begin{array}{l}\text { Dar sentido aos dados, encontrar padrões e } \\
\text { tirar conclusões no contexto da atividade } \\
\text { desenvolvida. }\end{array}$} \\
\hline \multicolumn{6}{|l|}{$\begin{array}{l}\text { Representar e organizar dados } \\
\text { apropriadamente por meio de gráficos, } \\
\text { tabelas, palavras ou imagens no contexto da } \\
\text { atividade desenvolvida. }\end{array}$} \\
\hline \multicolumn{6}{|l|}{$\begin{array}{l}\text { Dividir tarefas em partes menores e } \\
\text { gerenciáveis para resolver uma atividade } \\
\text { maior. }\end{array}$} \\
\hline \multicolumn{6}{|l|}{$\begin{array}{l}\text { Reduzir a complexidade de um problema } \\
\text { para focar na ideia principal. }\end{array}$} \\
\hline \multicolumn{6}{|l|}{$\begin{array}{l}\text { Seguir uma série de passos ordenados para } \\
\text { resolver um problema ou atingir algum } \\
\text { objetivo. }\end{array}$} \\
\hline \multicolumn{6}{|l|}{$\begin{array}{l}\text { Usar computadores ou máquinas para fazer } \\
\text { tarefas repetitivas ou tediosas durante o } \\
\text { desenvolvida de uma atividade especifica. }\end{array}$} \\
\hline \multicolumn{6}{|l|}{$\begin{array}{l}\text { Representar ou modelar um processo } \\
\text { relacionado à atividade desenvolvida. }\end{array}$} \\
\hline $\begin{array}{l}\text { Organizar recursos para, simultaneamente, } \\
\text { realizar tarefas para atingir um objetivo em } \\
\text { comum. }\end{array}$ & & & & & \\
\hline
\end{tabular}

Figura 2. Habilidades de PC analisadas no questionário

\subsection{Processo de coleta de dados}

O contato inicial com os participantes se deu no primeiro dia de aula da disciplina ESO mencionada do curso de LC da UFRPE. Tal disciplina foi ofertada nos dois semestres de 2016, havendo cinco estudantes matriculados no primeiro semestre e dois no segundo. Todos aceitaram participar do estudo de forma voluntária. 
VI Congresso Brasileiro de Informática na Educação (CBIE 2017)

Anais do XXIII Workshop de Informática na Escola (WIE 2017)

Havendo ciência dos objetivos, pelos participantes, seguiu-se com a entrega dos questionários, que foram respondidos em sala de aula. As respostas dadas pelos licenciandos em Computação, além de servirem para esta investigação, guiaram o planejamento da disciplina que teve como resultados: $i$ ) planejamento e regência de curso sobre pensamento computacional para professores da educação básica; ii) proposta de ementa de curso de desenvolvimento de jogos digitais para crianças; e iii) aplicativo de apoio à aprendizagem de Matemática, pela exploração de habilidades computacionais, para estudantes dos anos iniciais do ensino fundamental. Os resultados descritos nos itens $i$ e $i$ i foram alcançados no primeiro semestre de 2016; enquanto que o iii foi no segundo semestre do referido ano.

\subsection{Análise e síntese dos dados}

Técnicas da Teoria Fundamentada - Grounded Theory (CORBIN; STRAUSSET, 2007) foram usadas para codificar, categorizar e sintetizar os dados, sendo possível, ao fim do estudo, construir uma narrativa, na forma de teoria local, capaz de agregar valor às investigações no contexto dos cursos de Licenciatura em Computação.

Inicialmente foi feita a codificação aberta dos dados coletados, exceto das respostas à questão que buscava identificar as habilidades de pensamento computacional exploradas no dia a dia. Tal processo possibilitou a identificação de propriedades do fenômeno de interesse, as quais são chamadas de códigos ou categorias.

Códigos foram construídos à medida que a análise prosseguia e estavam relacionados a partes do texto. Em seguida, os códigos resultantes de cada questionário foram constantemente comparados com códigos resultantes do mesmo e dos demais questionários. A partir das comparações constantes, os códigos foram agrupados em categorias que representam a compreensão dos participantes sobre PC. A análise seguiu com o estabelecimento de relações entre as categorias formadas, sendo possível a criação da narrativa central, resultado do processo de síntese. Os resultados dessa tarefa são expostos e discutidos na seção seguinte.

\section{Resultados e discussões}

Nesta seção serão apresentados os resultados segmentados em suas partes: compreensão de PC e habilidades exploradas no dia a dia. Importante ressaltar que dos cinco participantes do estudo, referente à turma do primeiro semestre de 2016, um não tinha consciência do termo pensamento computacional, respondendo apenas à questão que tratava das habilidades de PC em seu cotidiano. Outro deixou de responder às questões que abordavam as estratégias pedagógicas e tecnologias que na visão dele poderiam ser adotadas nos processos de ensino e aprendizagem de PC. Este último caso também foi observado no questionário de um dos participantes do segundo semestre de 2016, que informou que não tinha conhecimento suficiente para opinar sobre os assuntos questionados.

\subsection{Entendimento sobre pensamento computacional}

A Figura 3 sumariza o relacionamento de fatores associados ao entendimento dos participantes deste estudo sobre PC. Como pode ser observado, na percepção dos licenciandos em Computação investigados, PC consiste na exploração de <fundamentos da Ciência da Computação>, de $<$ lógica computacional $>$ e <programação $>$ em conjunto com a exercitação do <pensamento crítico $>$. Tal achado está em consonância com a literatura da área por, principalmente, não associar o termo à utilização de tecnologia, em detrimento do ensino dos fundamentos da 
VI Congresso Brasileiro de Informática na Educação (CBIE 2017)

Anais do XXIII Workshop de Informática na Escola (WIE 2017)

Computação. Ressalta-se ainda que a <programação $>$ não foi percebida como única maneira para disseminação do PC, tendo ela efeito moderado nesse processo, quando trabalhada como meio para exploração de PC em sala de aula. Tal visão também é percebida no artigo seminal de Wing (2006) que defende que o ensino de PC não se restringe à programação.

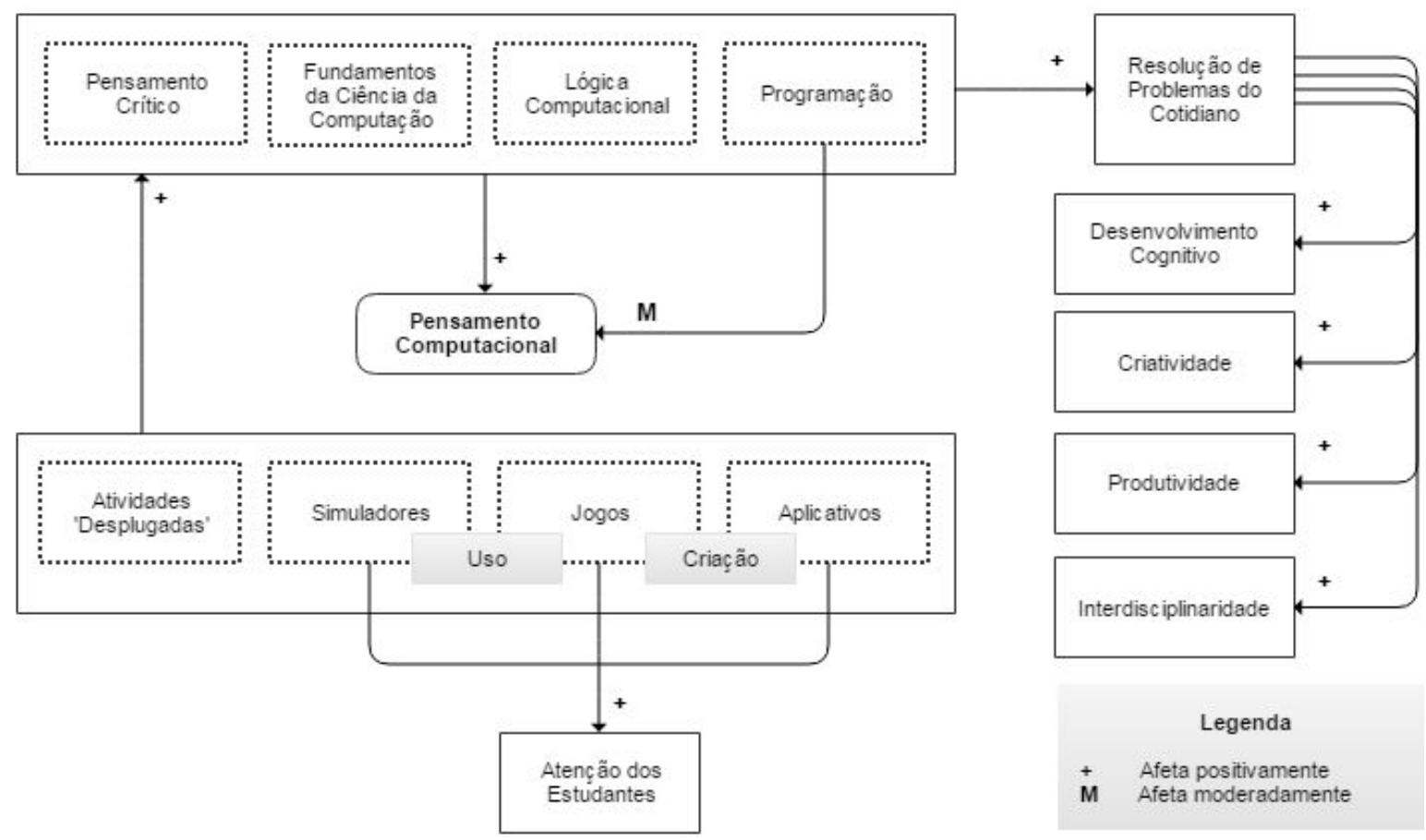

Figura 3. Modelo de alto nível sobre a compreensão de pensamento computacional

Os licenciandos investigados foram capazes de identificar diversas estratégias e recursos pedagógicos que podem apoiar os processos de ensino e aprendizagem de PC, dentre os quais pode-se mencionar <atividades desplugadas $>$, <jogos $>$, <simuladores $>$ e $<$ aplicativos $>$. Ao comparar tal resultado com trabalhos dispostos na literatura, um dos principais recursos identificados foi proposto por Bell et al. (2011): conjunto de atividades lúdicas, publicadas no livro Computer Science Unplugged. Tais atividades estimulam o pensamento computacional sem o uso do computador e são passíveis de aplicação em escolas carentes de recursos tecnológicos. No cenário brasileiro é possível identificar diversas ações que usaram atividades desplugadas nas escolas e obtiveram resultados positivos, como as reportadas em Ferreira et al. (2015).

$\mathrm{Na}$ visão dos participantes deste estudo, há maneiras diversificadas de aplicação de um mesmo tipo de recurso tecnológico em sala de aula. A depender do conteúdo a ser tratado, os $<$ jogos $>$, por exemplo, podem ser integrados às aulas de PC, podendo os estudantes fazerem $\langle$ uso $>$ desse recurso pela resolução de desafios propostos ou ainda construir conhecimento sobre conceitos computacionais à medida que se envolvem na <criação $>$ de seus próprios jogos.

Quanto ao $\langle u s o\rangle$, é possível identificar na literatura que jogos como Light Bot ${ }^{9}$ são passíveis de serem integrados às aulas de $\mathrm{PC}$. Em relação à <criação $>$, ferramentas que possibilitam a programação por meio do encaixe de blocos de comandos são usadas com recorrência, a exemplo de Scratch $^{10}$ e App Inventor ${ }^{11}$.

\footnotetext{
${ }^{9} \mathrm{https}: / /$ lightbot.com/

${ }^{10} \mathrm{https://scratch.mit.edu/}$

${ }^{11}$ https://appinventor.mit.edu/
} 
VI Congresso Brasileiro de Informática na Educação (CBIE 2017)

Anais do XXIII Workshop de Informática na Escola (WIE 2017)

Ainda, na percepção dos participantes a integração de <jogos>, <simuladores $>$ e $<$ aplicativos> pode focar a atenção dos estudantes sobre os conceitos computacionais ensinados, podendo repercutir positivamente sobre a aprendizagem.

O processo de <resolução de problemas do cotidiano > com a aplicação métodos e técnicas computacionais foi percebido como principal objetivo a ser alcançado em salas de aula de PC. Com isso, os futuros professores de Computação esperam contribuir para o <desenvolvimento cognitivo $>$ dos estudantes, estimular sua $<$ criatividade $>$ e aumentar sua $<$ produtividade $>$ em tarefas do dia a dia. Complementarmente, a resolução de problemas por meio do PC pode promover a <interdisciplinaridade> nas escolas, pela exploração de conceitos computacionais atrelada a conteúdos curriculares de outras áreas. Tal visão também está em conformidade com estudos sobre em Educação em Computação. Considerando a interdisciplinaridade do PC, por exemplo, Barcelos e Silveira (2013) investigaram de que maneira competências relacionadas à Matemática são mobilizadas por estudantes no processo de desenvolvimento do PC pela construção de jogos digitais.

\subsection{Habilidades exploradas no cotidiano}

Em relação às habilidades de PC que os professores reconhecem explorar na realização de tarefas do dia a dia, a sumarização dos dados é apresentada na Figura 4. Como pode ser observado, as habilidades associadas a Algoritmo, Automação e Abstração são usadas com maior recorrência. Coleta, Análise e Representação de Dados aparecem na sequência, sendo que esta última não teve a opção "sempre" assinalada pelos participantes.

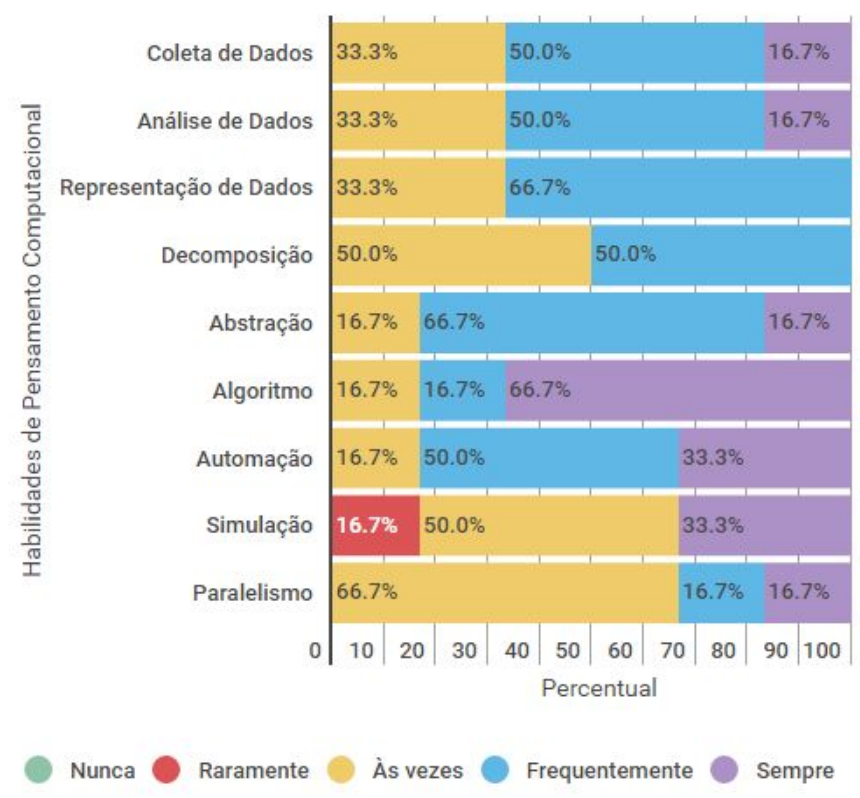

Figura 4. Habilidades de PC exploradas no cotidiano

Enquanto que Decomposição foi apontada como uma das habilidades de PC mais usadas pelos profissionais investigados no trabalho de Araújo et al. (2015), nesta pesquisa essa habilidade foi percebida como uma das menos exploradas pelos professores em tarefas cotidianas. Paralelismo e Simulação aparecem com um pouco mais de 33\%, somando os resultados assinalados com "sempre" e "frequentemente", sendo que Simulação foi apontada por um dos investigados como uma habilidade raramente usada em seu dia a dia. 
A partir de tais achados, alguns questionamentos podem feitos, dentre eles: $i$ ) como conceitos computacionais podem ser aplicados na resolução de problemas do cotidiano?; ii) como a formação do licenciado em Computação tem o ajudado a aplicar e a ensinar conceitos computacionais na resolução de problemas do dia a dia?; iii) que conceitos computacionais estão mais propensos de serem utilizados e percebidos por licenciados em Computação na resolução de problemas do cotidiano?.

Quanto ao item $i$, conceitos como iteração e eficiência podem ser tratados em uma operação matemática de multiplicação como $3 \times 4$. Esta pode ser resolvida pela decomposição e soma de $3+3+3+3$ ou ainda por $4+4+4$. Enquanto que na primeira opção há quatro iterações, na segunda há somente três, podendo ser considerada a mais eficiente. Assim como esse exemplo, vocábulos e símbolos que descrevem processos computacionais podem ser incorporados às salas de aula, podendo aumentar a capacidade de resolução de problemas dos estudantes.

Em relação ao item ii, é importante que os cursos de LC repensem seus projetos pedagógicos tendo em vista a importância da exploração de PC na educação básica. Além disso, é importante refletir sobre como o professor que está sendo formado é capaz de abordar o PC em sala de aula, considerando a diversidade de profissões dos estudantes que ainda estarão na educação básica. Abordar os fundamentos da Computação na escola, sem criar uma aversão à área, pode configurar-se um desafio, principalmente quando não há uma política pública que trate da inserção do PC no currículo escolar.

O item iii, sobre o uso e percepção de PC na resolução de problemas do dia a dia, pode estar associado à formação do licenciado em Computação. Se ela contempla os fundamentos da CC em seu currículo, mas o processo formativo não ocorre de maneira significativa, o licenciando pode fazer uso de conceitos computacionais em suas atividades cotidianas e não ter consciência disso. Por outro lado, se ele percebe a importância desses conceitos computacionais, poderá melhorar seu processo de sistematização ou organização de soluções de problemas, sejam elas profissionais ou do cotidiano.

\section{Considerações finais}

Neste trabalho um estudo de cunho qualitativo foi realizado objetivando identificar o entendimento de futuros professores de Computação sobre pensamento computacional. Nesse sentido, estudantes de um curso de Licenciatura em Computação responderam a um questionário e a partir da análise dos dados coletados identificou-se que a maioria deles foi capaz de definir PC, não havendo registros de associação do termo ao simples uso da tecnologia. Por outro lado, é importante ressaltar que um deles não respondeu ao questionamento, o que sugere que havia desconhecimento sobre termo, apesar desse participante ter cursado disciplinas que possibilitam a docência em Computação na educação básica. Neste caso, é importante comentar que no decorrer do semestre, na disciplina de ESO investigada, tal participante relatou ter vivenciado experiências de ensino de PC, não tendo ciência disso quando esta pesquisa foi realizada.

Boa parte dos participantes conseguiu identificar estratégias pedagógicas para o ensino de PC, tais como atividades desplugadas e uso/criação de jogos. Outros, no entanto, não forneceram sua visão sobre esse aspecto, o que pode tornar-se mais um desafio para o ensino de PC nas escolas. Ainda, os resultados sugerem que, em geral, os professores reconhecem a importância do ensino de PC, apontando como objetivo primordial a resolução de problemas do cotidiano, potencializando a criatividade dos estudantes e podendo também promover a 
VI Congresso Brasileiro de Informática na Educação (CBIE 2017)

Anais do XXIII Workshop de Informática na Escola (WIE 2017)

interdisciplinaridade no contexto escolar.

Quanto às habilidades de PC exploradas pelos participantes do estudo na resolução de problemas do dia a dia, Algoritmo, Automação e Abstração são sempre ou frequentemente usadas. Por outro lado, Paralelismo e Simulação foram apontadas como as menos exploradas. Este resultado pode levar o curso investigado a refletir sobre como o PC tem sido tratado em seu currículo e que estratégias têm sido usadas para tornar a aprendizagem dos estudantes mais significativa, tendo os licenciandos clareza dos conteúdos e de sua aplicação na resolução de problemas.

Não há a pretensão de generalizar os resultados obtidos, até mesmo pela natureza do estudo realizado. Espera-se, contudo, contribuir com a discussão sobre o professor de Computação que está sendo formado e o seu papel na disseminação do PC nas escolas brasileiras. Como trabalhos futuros, almeja-se replicar o estudo e acompanhar a trajetória de aprendizagem dos estudantes buscando identificar, além dos aspectos já considerados neste trabalho, a confiança para o ensino de PC, considerando desafios na formação desse profissional e também no contexto de seu campo de atuação.

\section{Referências}

Araújo, A. L. S. O.; Andrade, W. L.; Guerrero, D. D. S. (2015). "Pensamento Computacional sob a visão dos profissionais da computação: uma discussão sobre conceitos e habilidades". In: Anais dos Workshops do IV CBIE.

Barcelos, T. S.; Silveira, I. F. (2013). "Relações entre o Pensamento Computacional e a Matemática através da construção de Jogos Digitais”. In: Anais do XII SBGames.

Bell, T.; Witten, I, H.; Fellows, M. (2011). Computer Science Unplugged: Ensinando Ciência da Computação sem o uso do computador. Tradução coord. por Luciano Porto Barreto.

Bower, M.; Lister, R.; Mason, R.; Highfield, K.; Wood, L. (2015). “Teacher conceptions of computational thinking: implications for policy and practice". Australian Journal of Education.

CSTA. (2005). The New Educational Imperative: Improving High School Computer Science Education. Final Report of the CSTA. Curriculum Improvement Task Force. ACM.

CSTA - Computer Science Teacher Association (2011). CSTA K-12 Computer Science Standards. CSTA Standards Task Force. ACM - Association for Computing Machinery.

Corbin, J.; Strauss, A. (2007). Basics of Qualitative Research: Techniques and Procedures for Developing Grounded Theory. SAGE Publications, Inc; 3rd edition.

Ferreira, A. C. C., Melhor, A., Barreto, J. S., Paiva, L. F., Matos, E. (2015). "Experiência Prática Interdisciplinar do Raciocínio Computacional em Atividades de Computação Desplugada na Educação Básica”. In: Anais do XXI WIE, p. 256-265.

Ministério da Educação (MEC), Brasil. Base Nacional Comum Curricular. Disponível em $<\mathrm{http}$ ///basenacionalcomum.mec.gov.br/images/BNCC_publicacao.pdf $>$. Acesso em 01 de jun. 2017

Nunes, D. J. (2008). Licenciatura em Computação. Jornal da Ciência / Notícias nº3522 de 30 de maio de 2008. Acesso em 01 de jun. 2017. Disponível em $<$ https://www.lncc.br/lncc/noticias.php?idt_noticia=209>.

ISTE; CSTA; NSF. (2011). Computational thinking: leadership toolkit. First Edition.

Wing, J. M. (2006). “Computational thinking”. Communications of the ACM, v. 49, n. 3, p. 33-35. 